\title{
Azurin-derived peptides: Comparison of nickel and copper-binding properties
}

\author{
Debanjana Das and Sri Rama Koti Ainavarapu* \\ Department of Chemical Sciences, Dr. Homi Bhabha Road, Colaba, \\ Tata Institute of Fundamental Research, Mumbai-400005
}

Key words: Azurin, Nickel-binding, Charge transfer, Quenching, Peptide mimicking

*Corresponding author:

Email for correspondence: koti@tifr.res.in 


\section{Materials and methods}

\section{Solid phase peptide synthesis}

Peptides were prepared using Fast Flow Solid Phase Peptide Synthesis as described in a previous report. ${ }^{1}$ Peptides were synthesized with the help of a fast-flow solid phase peptide synthesizer provided and protocol optimised by Prof. Ankona Datta and her lab members (Mr. Akshay Kapadia) in TIFR, Mumbai. Four peptides were synthesised with the following sequences (Table S1).

\section{Table S1:}

\begin{tabular}{|c|c|c|c|}
\hline Peptide & Label & Sequence & Mol. Mass (Da) \\
\hline AzurinWT (111-123) 13 residues & AzuWT13aa & FCETFPG프SALMKG & 1394 \\
\hline AzurinWT (111-121) 11 residues & AzuWT11aa & FCETFPG $\underline{H} S A L \underline{M}$ & 1210 \\
\hline AzurinF114W 13 residues & AzuF114W & FC彑TWPG $\underline{H} S A L \underline{M K G}$ & 1433 \\
\hline AzurinF111W 13 residues & AzuF111W & WCTTFPG $\underline{H} S A L M K G$ & 1433 \\
\hline
\end{tabular}

Table S1. Azurin peptides used in the study. The three copper ligands (Cys112, His117, and Met121) are underlined and the Trp mutations are shown in bold.

\section{Absorbance experiments}

Absorbance data were recorded on a Specord-205 UV-Vis spectrophotometer. Peptide concentrations in solution were estimated using tryptophan or phenylalanine absorbance, whichever was present in the peptides. For wild-type peptides, the molar extinction coefficient $(\epsilon)$ of phenylalanine was taken as $195 \mathrm{M}^{-1} \mathrm{~cm}^{-1}$ at $257 \mathrm{~nm} \cdot{ }^{2,3}$ For tryptophan-containing peptides, $\epsilon$ of tryptophan was taken as $5579 \mathrm{M}^{-1} \mathrm{~cm}^{-1}$ at $278 \mathrm{~nm}^{2,3}$ Peptide concentrations were kept at $\sim 100 \mu \mathrm{M}$ for all experiments and $\mathrm{Cu}^{2+}$ and $\mathrm{Ni}^{2+}$ were added in the form of $\mathrm{CuSO}_{4}$ and $\mathrm{NiSO}_{4}$ supplied by Qualigens Fine Chemicals. Stock solution concentrations of $\mathrm{CuSO}_{4}$ and $\mathrm{NiSO}_{4}$ were $3 \mathrm{mM}$. For all titration experiments, the $\mathrm{Ni}^{2+}$ concentration was increased by $10 \mu \mathrm{M}$ or $20 \mu \mathrm{M}$. The path length of the solution used was $1 \mathrm{~cm}$. All experiments were done at room temperature $\left(\sim 25^{\circ} \mathrm{C}\right)$ in ambient 
conditions in $5 \mathrm{mM}$ phosphate buffer ( $\mathrm{pH}$ 7.4). Buffer composition was sodium dihydrogen phosphate, disodium hydrogen phosphate, and sodium chloride.

\section{Dissociation Constant $\left(\mathrm{K}_{\mathrm{d}}\right)$ Calculation of 1:1 Ni ${ }^{2+}$ :peptide complexes}

From preliminary absorption, $\mathrm{CD}$, and fluorescence titrations, the $\mathrm{Ni}^{2+}$-peptide complex is evidenced to form in a 1:1 stoichiometry. The apparent dissociation constants $\left(\mathrm{K}_{\mathrm{d}}\right)$ for the 1:1 $\mathrm{Ni}^{2+}$ :peptide complexes were determined from a plot of observed absorbance ratios in the presence and absence of metal ion $\left(\mathrm{A} / \mathrm{A}_{0}\right)$ at $270 \mathrm{~nm}$ versus total $\left[\mathrm{Ni}^{2+}\right]$ concentration. The value of $\mathrm{K}_{\mathrm{d}}$ was calculated by fitting the data to the following equation. ${ }^{4}$

$$
\frac{A}{A_{0}}=1+\left(A_{\max }-A_{0}\right) \times \frac{\left\{\left([P]_{t}+\left[N i^{2+}\right]_{t}+K_{d}\right)-\sqrt{\left([P]_{t}+\left[N i^{2+}\right]_{t}+K_{d}\right)^{2}-4 \times[P]_{t} \times\left[N i^{2+}\right]_{t}}\right\}}{\left(2 \times[P]_{t} \times A_{0}\right)}
$$

In this equation, $A_{\max }$ is the maximum value of absorbance attained in the titration curve and $A_{0}$ is the absorbance value of the respective peptides without addition of $\mathrm{Ni}^{2+}$-ion.

$[P]_{t}$ is the total concentration of the respective peptides used during titration.

$\left[N i^{2+}\right]_{t}$ is the total $\mathrm{Ni}^{2+}$-ion concentration added at a particular titration point.

\section{Circular dichroism experiments}

The secondary structure of the peptides was probed using circular dichroism (CD). The CD data was recorded on a Jasco J-1500 spectrometer. Peptide concentrations for CD experiments were $\sim 100 \mu \mathrm{M}$ and $\mathrm{Ni}^{2+}$ was added in the form of $\mathrm{NiSO}_{4}$. Stock solution concentration of $\mathrm{NiSO}_{4}$ was 3 $\mathrm{mM}$. The path length used was $1 \mathrm{~mm}$. The averages of 3 spectra were taken. All experiments were done at room temperature $\left(\sim 25^{\circ} \mathrm{C}\right)$ in $5 \mathrm{mM}$ phosphate buffer $(\mathrm{pH} 7.4)$.

\section{Steady-state fluorescence experiments}

Steady-state fluorescence experiments were performed to study intensity of tryptophan fluorescence quenching on $\mathrm{Ni}^{2+}$ addition. These measurements were done on a SPEX fluorolog $\mathrm{T}$ format fluorimeter (Horiba Jobin Yvon). The excitation wavelength used was $295 \mathrm{~nm}$. The averages of 5 spectra were taken. Peptide concentrations were kept at $\sim 100 \mu \mathrm{M}$ concentration and $\mathrm{Ni}^{2+}$ was 
added from a stock solution of $3 \mathrm{mM} \mathrm{NiSO}$. All experiments were done at room temperature $\left(\sim 25^{\circ} \mathrm{C}\right)$ in $5 \mathrm{mM}$ phosphate buffer $(\mathrm{pH} 7.4)$.

\section{Time resolved fluorescence experiments}

Time-resolved fluorescence of tryptophan was determined using time correlated single photon counting (TSCPC) technique. The TCSPC data were recorded using a home-built setup that utilizes a pico-second dye laser ${ }^{5,6}$. The instrument response function (IRF) was $\sim 80$ ps. Tryptophan was excited at $295 \mathrm{~nm}$ and emission was collected at $360 \mathrm{~nm}$. Fluorescence decay data were collected such that the peak count was $>10000$. Analysis of TCSPC data was done as described previously ${ }^{6}$. Fluorescence decays were fitted to three exponentials by minimizing $\chi_{R}^{2}$. Mean fluorescence lifetime $\left(\tau_{\mathrm{m}}\right)$ and amplitude-weighted lifetime $\left(\tau_{\mathrm{a}}\right)$ were estimated using the

expressions, $\tau_{m}=\frac{\sum \alpha_{i} \tau_{i}^{2}}{\Sigma \alpha_{i} \tau_{i}}$ and $\tau_{a}=\frac{\Sigma \alpha_{i} \tau_{i}}{\Sigma \alpha_{i}}$, respectively. Here $\alpha_{i}$ and $\tau_{i}$ are amplitude and lifetime, respectively, of the $i^{\text {th }}$ component in the decay analysis. 


\section{Figure S1.}

$\begin{array}{lllll}1 & 11 & 21 & 31 & 41 \\ \text { AECSVDIQGN } & \text { DQMQFNTNAI } & \text { TVDKSCKQFT } & \text { VNLSHPGNLP } & \text { KNVMGHNWVI } \\ 51 & 61 & 71 & 81 & 91 \\ \text { STAADMQGVV } & \text { TDGMASGLDK } & \text { DYLKPDDSRV } & \text { IAHTKLIGSG } & \text { EKDSVTFDVS } \\ 101 & 111 & 121 & & \\ \text { KLKEGEQYMS } & \text { FCTFPGHSAL } & \text { MKG } & \text { TLTLK } & \end{array}$

Figure S1. Protein sequence for azurin derived from Pseudomonas aeruginosa, with the 13 amino acid peptide sequence used for studies highlighted. Copper/nickel binding residues are also highlighted in blue. 
Figure S2.
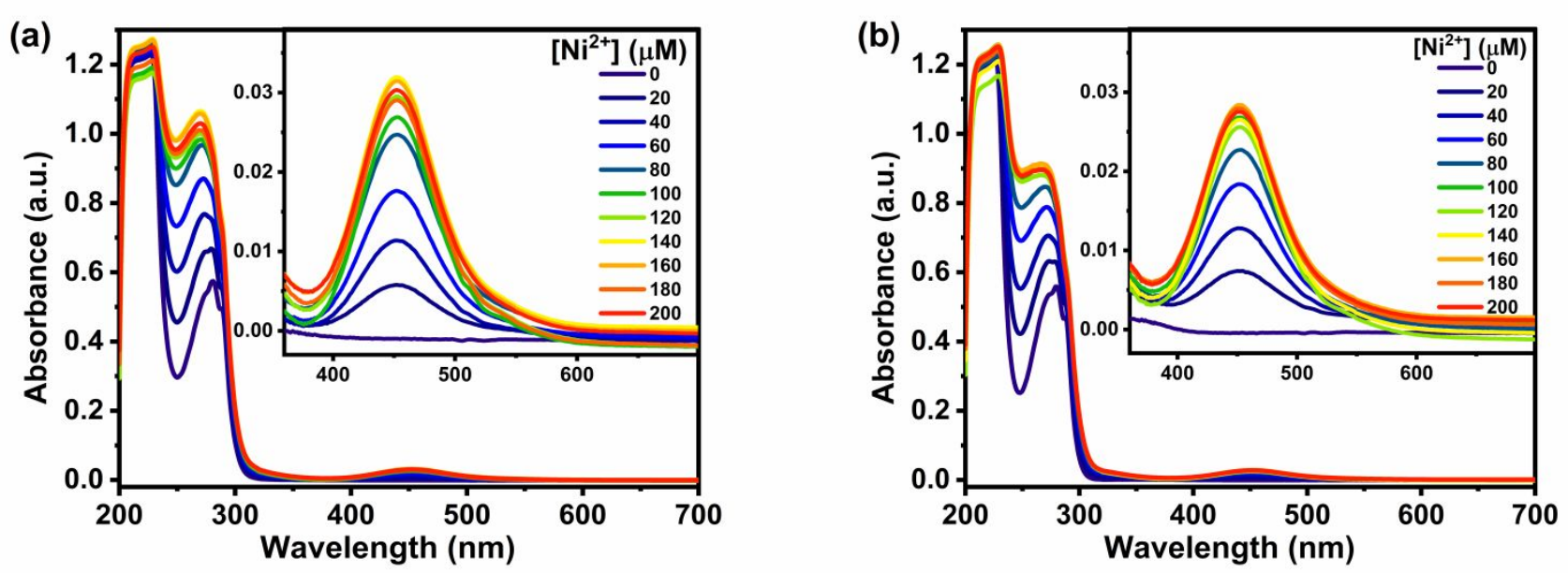

Figure S2. Absorption spectra for (a) AzuF114W and (b) AzuF111W upon $\mathrm{Ni}^{2+}$ titration. (Peptide concentrations kept were $100 \mu \mathrm{M}$ and buffer used was $5 \mathrm{mM}$ phosphate buffer, $\mathrm{pH}$ 7.4) 
Figure S3.
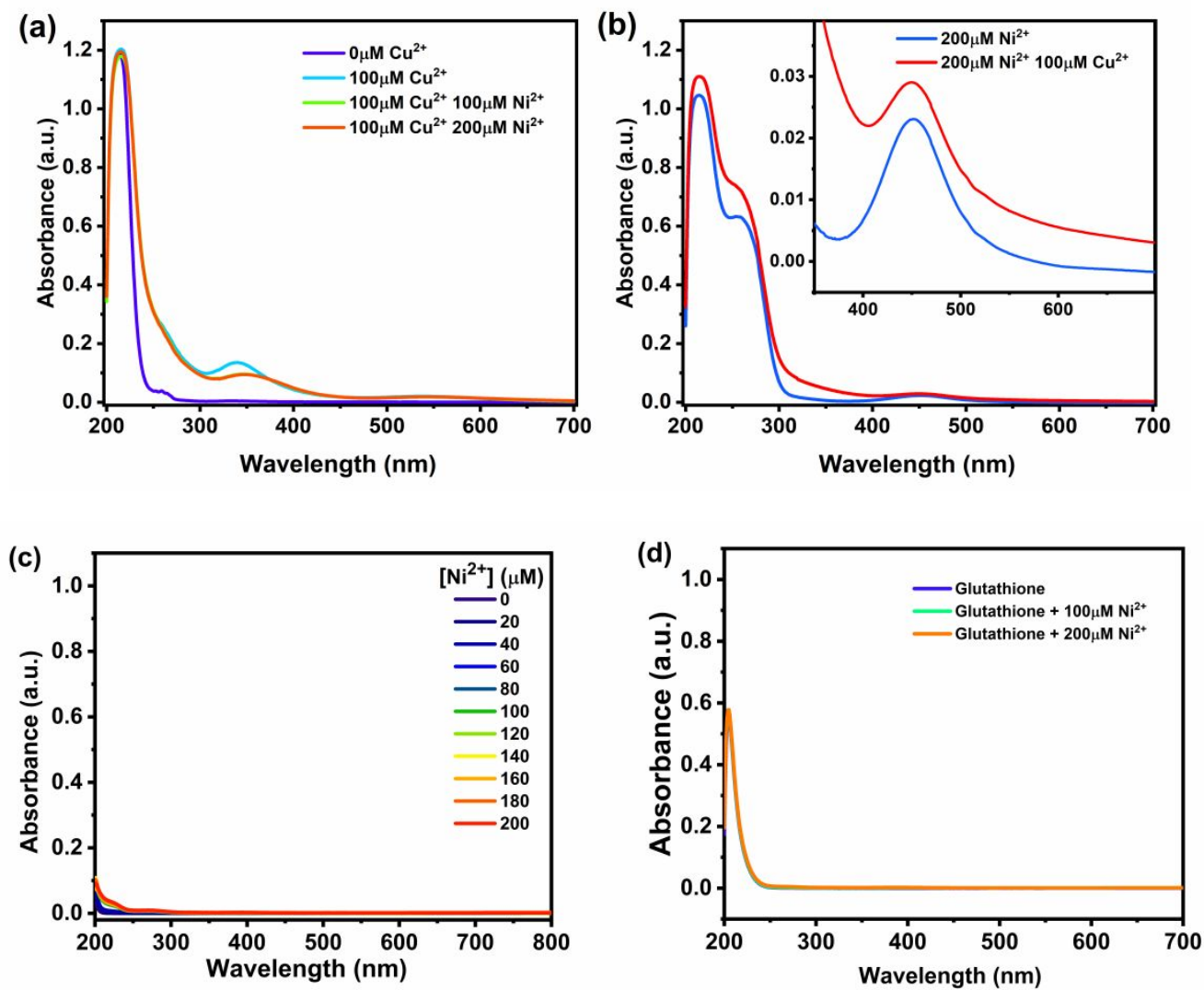

(e)

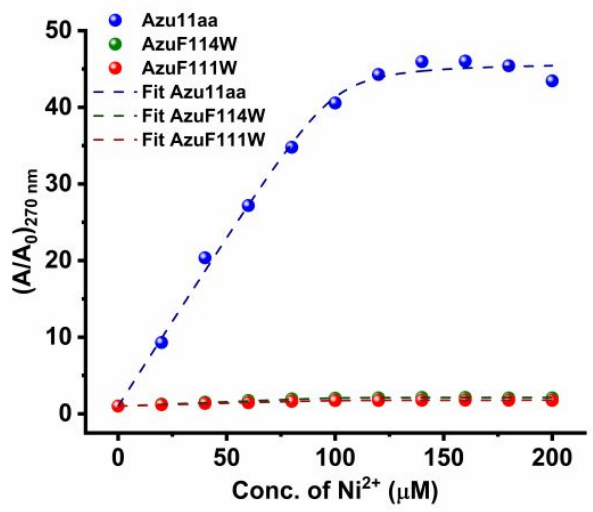

Figure S3. (a) Absorption spectra for AzuWT13aa on $\mathrm{Cu}^{2+}$ addition followed by $\mathrm{Ni}^{2+}$ addition. (b) Absorption spectra for AzuWT13aa on $\mathrm{Ni}^{2+}$ addition followed by $\mathrm{Cu}^{2+}$ addition. (Inset) Zoomed in spectra. (Peptide concentrations kept were $\sim 100 \mu \mathrm{M}$ and buffer used was $5 \mathrm{mM}$ phosphate buffer, $\mathrm{pH}$ 7.4). (c) Blank absorption spectra for $\mathrm{Ni}^{2+}$ titration with $5 \mathrm{mM}$ phosphate buffered saline. (d) Absorption spectra for $\mathrm{Ni}^{2+}$ titration with $5 \mathrm{mM}$ phosphate buffered saline in presence of $100 \mu \mathrm{M}$ glutathione. (e) Plot of observed absorbance ratios in the presence and absence of metal ion $\left(\mathrm{A} / \mathrm{A}_{0}\right)$ at $270 \mathrm{~nm}$ versus total $\left[\mathrm{Ni}^{2+}\right]$ concentration for $\mathrm{Ni}^{2+}$-complexes of AzuWT11aa, AzuF114W, and AzuF111W. (Fits to the data are according to the equation given above.) 
Figure S4.

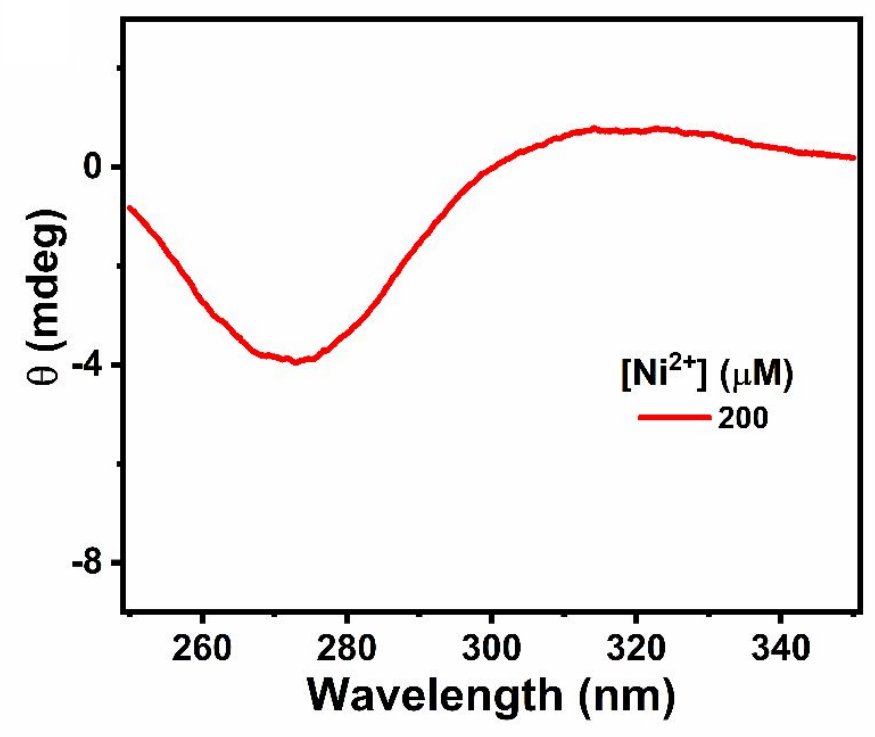

Figure S4. CD spectra of AzuWT11aa titrated with $\mathrm{Ni}^{2+}$ in the range $250-350 \mathrm{~nm}$ (CT absorbing region). (Peptide concentration kept was $100 \mu \mathrm{M}$ and buffer used was $5 \mathrm{mM}$ phosphate buffer, $\mathrm{pH}$ 7.4) 
Figure S5.
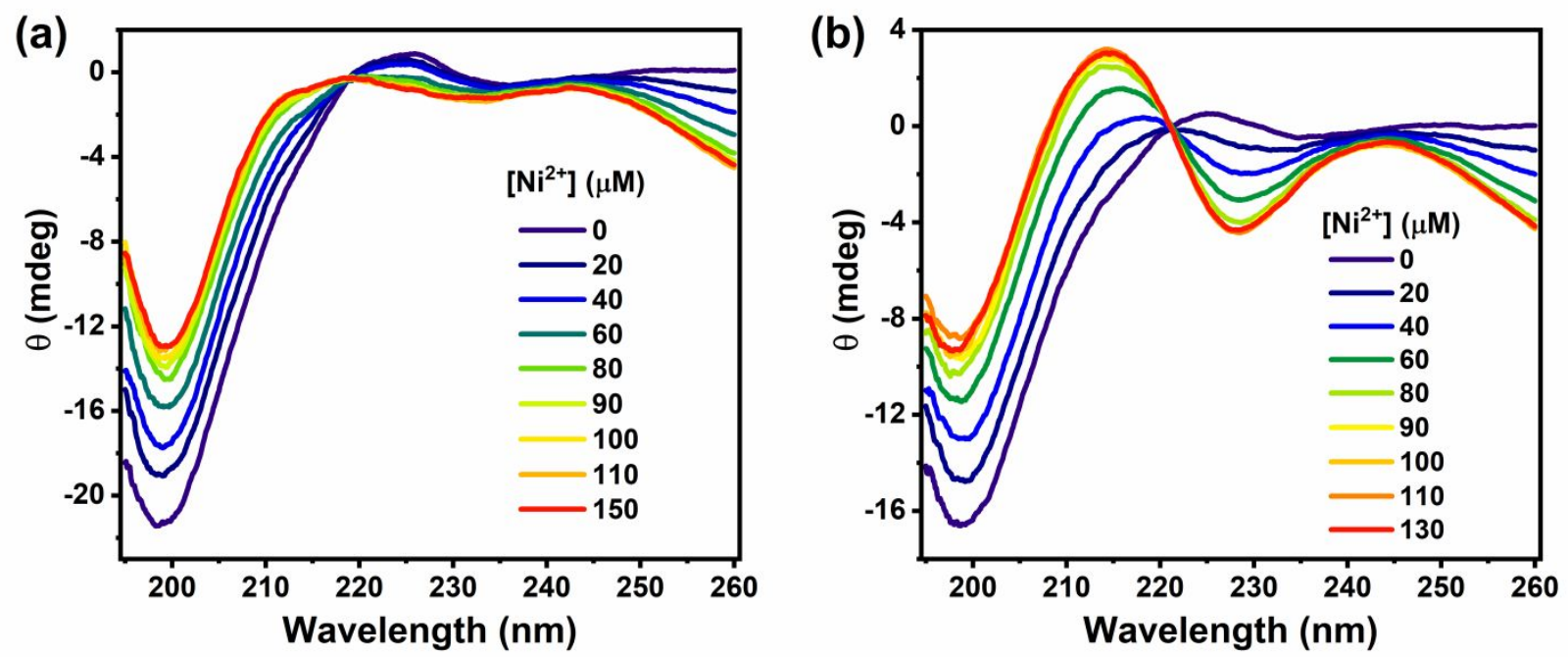

Figure S5. CD spectra for (a) AzuF114W and (b) AzuF111W upon $\mathrm{Ni}^{2+}$ titration. (Peptide concentrations kept were $100 \mu \mathrm{M}$ and buffer used was $5 \mathrm{mM}$ phosphate buffer, $\mathrm{pH}$ 7.4) 
Figure S6.
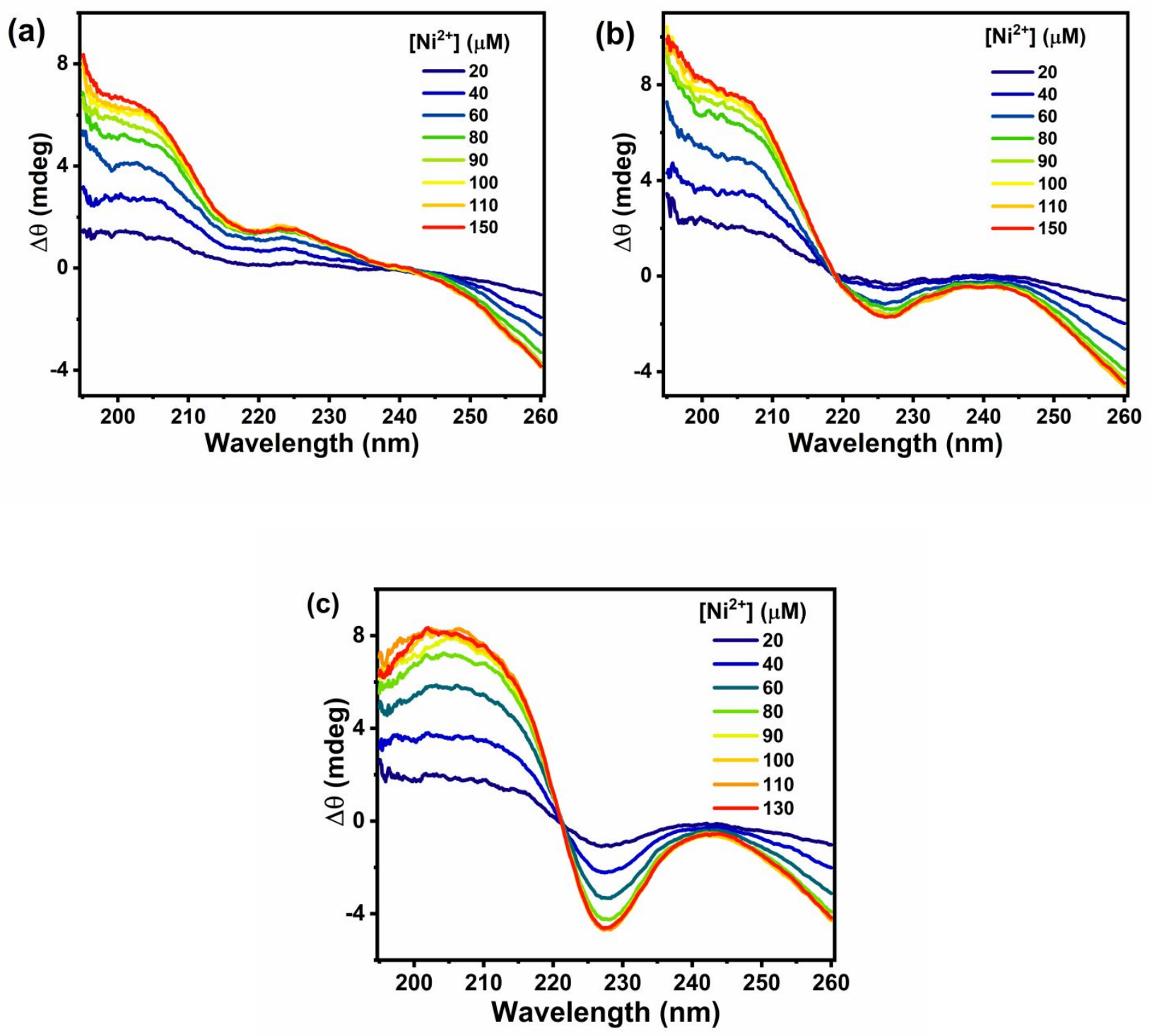

Figure S6. Difference CD spectra for (a) AzuWT11aa (b) AzuF114W and (c) AzuF111W upon $\mathrm{Ni}^{2+}$ titration. Difference $\mathrm{CD}$ is the $\mathrm{CD}$ spectra from nickel titration experiment subtracted with the spectrum at $0 \mu \mathrm{M}\left[\mathrm{Ni}^{2+}\right]$ for each peptide. (Peptide concentrations kept were $100 \mu \mathrm{M}$ and buffer used was $5 \mathrm{mM}$ phosphate buffer, $\mathrm{pH}$ 7.4) 
Figure S7.
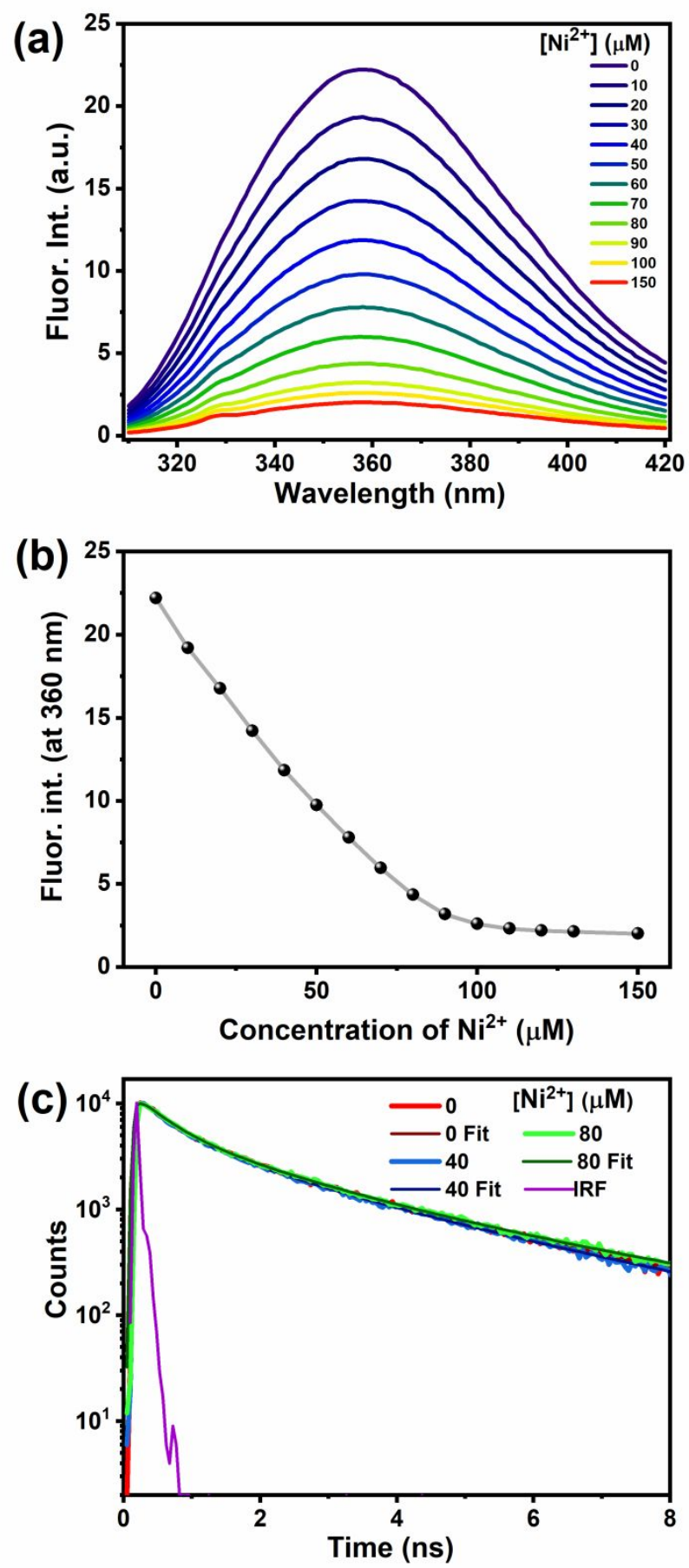

Figure S7. Fluorescence studies on the tryptophan peptide AzuF111W. (a) Steady-state fluorescence spectra of the peptide showing quenching upon $\mathrm{Ni}^{2+}$ titration. (b) Fluorescence vs $\left[\mathrm{Ni}^{2+}\right]$ at $360 \mathrm{~nm}$ from the data shown in (a). (c) Representative time-resolved fluorescence data along with the instrument-response function and multi-exponential fits. (Peptide concentrations kept were $100 \mu \mathrm{M}$ and buffer used was $5 \mathrm{mM}$ phosphate buffer, $\mathrm{pH}$ 7.4) 
Figure S8.
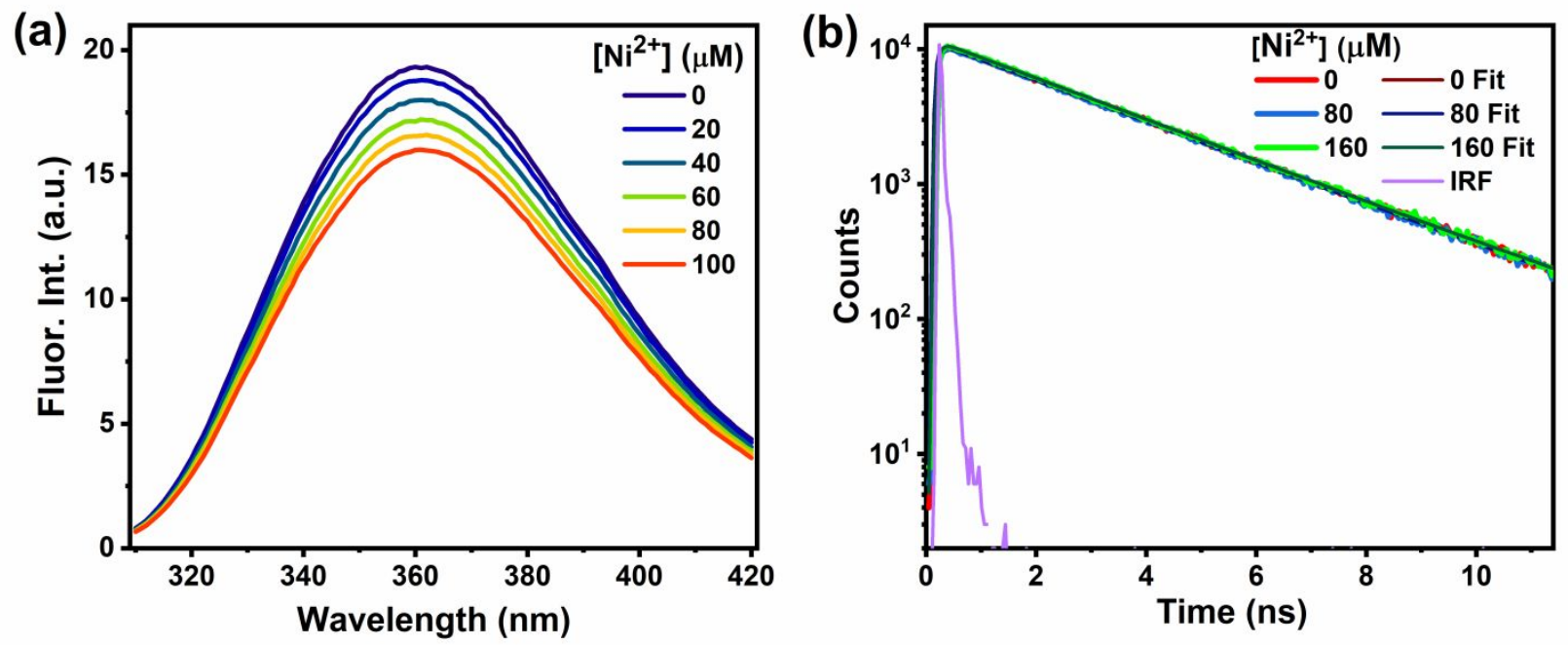

Figure S8. Fluorescence studies on NATA (a) Steady-state fluorescence spectra of N-acetyl tryptophanamide (NATA) showing quenching upon $\mathrm{Ni}^{2+}$ titration. (b) Representative time-resolved fluorescence data along with the instrument-response function and mono-exponential fits. (NATA concentration kept was $100 \mu \mathrm{M}$ and buffer used was $5 \mathrm{mM}$ phosphate buffer, $\mathrm{pH}$ 7.4) 
Figure S9.
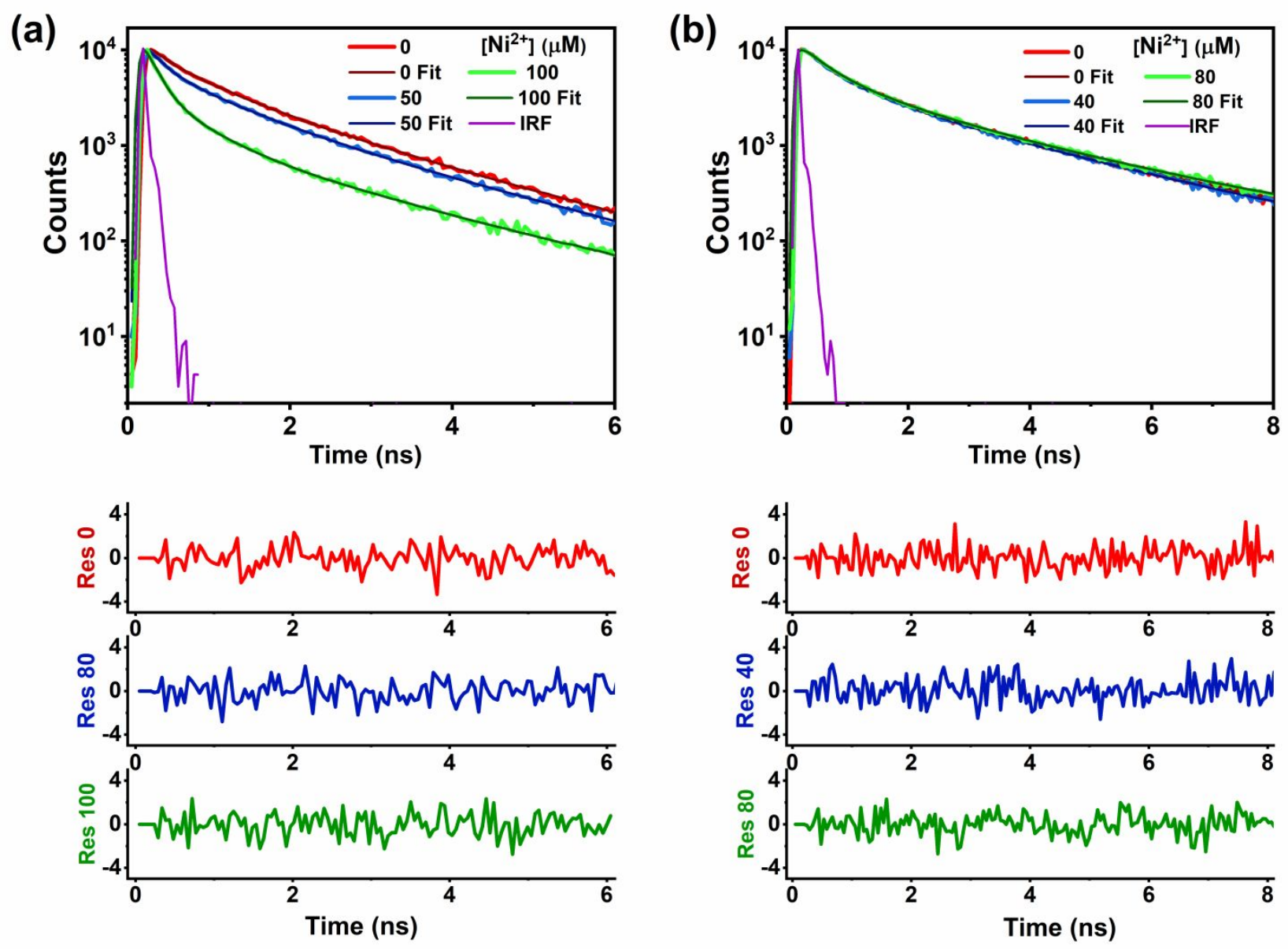

Figure S9. (a) Representative time-resolved fluorescence data along with the instrument-response function and multi-exponential fits for AzuF114W quenching at different $\left[\mathrm{Ni}^{2+}\right]$. The residuals for the corresponding fits are shown below. (b) Representative time-resolved fluorescence data along with the instrument-response function and multi-exponential fits for AzuF $111 \mathrm{~W}$ quenching at different $\left[\mathrm{Ni}^{2+}\right]$. (The residuals of the corresponding fits are shown below.) 
Table S2. Representative multi-exponential fits for the fluorescence decay traces of peptide with tryptophan (AzuF114W).

\begin{tabular}{|c|c|c|c|c|c|c|c|c|c|}
\hline $\begin{array}{c}\text { Conc. of } \mathrm{Ni}^{2+} \\
(\mu \mathrm{M})\end{array}$ & $\tau_{1}$ & $\alpha_{1}$ & $\tau_{2}$ & $\alpha_{2}$ & $\tau_{3}$ & $\alpha_{3}$ & $\tau_{\mathrm{a}}$ & $\tau_{m}$ & $\chi^{2}$ \\
\hline \multirow[t]{2}{*}{0} & 0.21 & 0.36 & 0.92 & 0.43 & 2.12 & 0.21 & 0.92 & 1.44 & 1.10 \\
\hline & 0.16 & 0.33 & 0.73 & 0.36 & 1.88 & 0.31 & 0.90 & 1.44 & 1.05 \\
\hline \multirow[t]{2}{*}{10} & 0.17 & 0.39 & 0.82 & 0.34 & 1.92 & 0.27 & 0.86 & 1.43 & 1.00 \\
\hline & 0.26 & 0.41 & 0.92 & 0.33 & 1.99 & 0.26 & 0.93 & 1.44 & 1.04 \\
\hline \multirow[t]{2}{*}{20} & 0.14 & 0.39 & 0.74 & 0.36 & 1.91 & 0.25 & 0.80 & 1.40 & 1.05 \\
\hline & 0.23 & 0.41 & 0.84 & 0.33 & 1.96 & 0.26 & 0.88 & 1.42 & 1.05 \\
\hline \multirow[t]{2}{*}{30} & 0.19 & 0.43 & 0.84 & 0.35 & 2.01 & 0.22 & 0.82 & 1.41 & 0.95 \\
\hline & 0.16 & 0.39 & 0.68 & 0.33 & 1.86 & 0.28 & 0.81 & 1.40 & 1.04 \\
\hline \multirow[t]{2}{*}{40} & 0.26 & 0.50 & 1.10 & 0.34 & 2.16 & 0.16 & 0.85 & 1.40 & 0.99 \\
\hline & 0.16 & 0.43 & 0.78 & 0.35 & 1.97 & 0.22 & 0.78 & 1.39 & 0.91 \\
\hline \multirow[t]{2}{*}{50} & 0.23 & 0.52 & 1.01 & 0.33 & 2.25 & 0.15 & 0.79 & 1.42 & 1.01 \\
\hline & 0.16 & 0.48 & 0.73 & 0.31 & 1.93 & 0.21 & 0.71 & 1.35 & 1.03 \\
\hline \multirow[t]{2}{*}{60} & 0.20 & 0.56 & 1.02 & 0.33 & 2.49 & 0.11 & 0.72 & 1.45 & 1.01 \\
\hline & 0.15 & 0.52 & 0.68 & 0.29 & 1.92 & 0.19 & 0.64 & 1.32 & 1.00 \\
\hline \multirow[t]{2}{*}{70} & 0.19 & 0.60 & 0.74 & 0.25 & 2.06 & 0.15 & 0.61 & 1.31 & 0.95 \\
\hline & 0.15 & 0.61 & 0.70 & 0.25 & 2.07 & 0.14 & 0.56 & 1.32 & 0.90 \\
\hline \multirow[t]{2}{*}{80} & 0.14 & 0.65 & 0.61 & 0.22 & 1.92 & 0.13 & 0.47 & 1.21 & 1.09 \\
\hline & 0.12 & 0.66 & 0.53 & 0.23 & 1.91 & 0.11 & 0.41 & 1.16 & 1.09 \\
\hline \multirow[t]{2}{*}{90} & 0.10 & 0.68 & 0.42 & 0.23 & 1.81 & 0.09 & 0.33 & 1.04 & 0.90 \\
\hline & 0.14 & 0.73 & 0.54 & 0.19 & 1.95 & 0.08 & 0.36 & 1.04 & 1.15 \\
\hline \multirow[t]{2}{*}{100} & 0.12 & 0.72 & 0.49 & 0.21 & 1.88 & 0.07 & 0.32 & 0.96 & 0.87 \\
\hline & 0.11 & 0.63 & 0.35 & 0.28 & 1.71 & 0.09 & 0.32 & 0.95 & 1.06 \\
\hline
\end{tabular}


Table S3. Representative multi-exponential fits for the fluorescence decay traces of peptide with tryptophan (AzuF111W).

\begin{tabular}{cccccccccc}
\hline $\begin{array}{c}\text { Conc. of } \mathbf{~ N i}^{2+} \\
(\mu \mathrm{M})\end{array}$ & $\tau_{1}$ & $\alpha_{1}$ & $\tau_{2}$ & $\alpha_{2}$ & $\tau_{3}$ & $\alpha_{3}$ & $\tau_{\mathrm{a}}$ & $\tau_{\mathrm{m}}$ & $\chi^{2}$ \\
\hline $\mathbf{0}$ & 0.30 & 0.38 & 1.02 & 0.36 & 3.07 & 0.26 & $\mathbf{1 . 2 8}$ & $\mathbf{2 . 2 3}$ & 1.03 \\
\hline $\mathbf{1 0}$ & 0.40 & 0.45 & 1.26 & 0.31 & 3.19 & 0.24 & $\mathbf{1 . 3 4}$ & $\mathbf{2 . 2 5}$ & 1.10 \\
\hline $\mathbf{2 0}$ & 0.33 & 0.40 & 1.08 & 0.35 & 3.13 & 0.25 & $\mathbf{1 . 2 9}$ & $\mathbf{2 . 2 4}$ & 1.12 \\
\hline & 0.37 & 0.40 & 1.12 & 0.35 & 3.17 & 0.25 & $\mathbf{1 . 3 3}$ & $\mathbf{2 . 2 6}$ & 1.13 \\
\hline $\mathbf{3 0}$ & 0.38 & 0.45 & 1.25 & 0.34 & 3.27 & 0.21 & $\mathbf{1 . 2 8}$ & $\mathbf{2 . 2 2}$ & 0.96 \\
\hline $\mathbf{4 0}$ & 0.36 & 0.43 & 1.17 & 0.34 & 3.23 & 0.23 & $\mathbf{1 . 3 0}$ & $\mathbf{2 . 2 5}$ & 1.10 \\
\hline $\mathbf{5 0}$ & 0.28 & 0.40 & 1.05 & 0.37 & 3.14 & 0.23 & $\mathbf{1 . 2 2}$ & $\mathbf{2 . 2 1}$ & 0.95 \\
\hline $\mathbf{6 0}$ & 0.38 & 0.44 & 1.15 & 0.32 & 3.14 & 0.24 & $\mathbf{1 . 2 9}$ & $\mathbf{2 . 2 1}$ & 1.11 \\
\hline $\mathbf{8 0}$ & 0.47 & 0.45 & 1.16 & 0.32 & 3.12 & 0.23 & $\mathbf{1 . 2 6}$ & $\mathbf{2 . 1 8}$ & 1.10 \\
\hline & 0.34 & 0.37 & 1.02 & 0.38 & 3.07 & 0.25 & $\mathbf{1 . 2 8}$ & $\mathbf{2 . 1 8}$ & 0.97 \\
\hline & 0.44 & 0.51 & 1.52 & 0.31 & 3.43 & 0.18 & $\mathbf{1 . 3 1}$ & $\mathbf{2 . 2 3}$ & 1.06 \\
\hline & 0.32 & 0.46 & 1.21 & 0.32 & 3.24 & 0.22 & $\mathbf{1 . 2 9}$ & $\mathbf{2 . 2 2}$ & 1.02 \\
\hline & 0.48 & 0.51 & 1.56 & 0.31 & 3.53 & 0.18 & $\mathbf{1 . 3 6}$ & $\mathbf{2 . 2 8}$ & 1.11 \\
\hline & 0.41 & 0.48 & 1.34 & 0.32 & 3.44 & 0.20 & $\mathbf{1 . 3 1}$ & $\mathbf{2 . 3 0}$ & 1.13 \\
\hline & 0.43 & 1.23 & 0.37 & 3.51 & 0.20 & $\mathbf{1 . 3 0}$ & $\mathbf{2 . 3 6}$ & 1.00 \\
\hline & 0.49 & 1.54 & 0.35 & 3.96 & 0.16 & $\mathbf{1 . 3 9}$ & $\mathbf{2 . 4 7}$ & 1.08 \\
\hline & & & 1.10 & 0.39 & 3.29 & 0.22 & $\mathbf{1 . 2 8}$ & $\mathbf{2 . 2 6}$ & 0.97 \\
\hline
\end{tabular}




\section{References-}

(1) Das, D.; Mitra, S.; Kumar, R.; Banerjee, S.; Koti Ainavarapu, S. R. Copper-Induced Spectroscopic and Structural Changes in Short Peptides Derived from Azurin. Arch. Biochem. Biophys. 2020, 687, 108388.

(2) Dixon, J. M.; Taniguchi, M.; Lindsey, J. S. PhotochemCAD 2: A Refined Program with Accompanying Spectral Databases for Photochemical Calculations. Photochem. Photobiol. 2005, $81(1), 212$.

(3) Du, H.; Fuh, R. C. A.; Li, J.; Corkan, L. A.; Lindsey, J. S. PhotochemCAD++: A ComputerAided Design and Research Tool in Photochemistry. Photochem. Photobiol. 1998, 68 (2), $141-142$.

(4) Bakthavatsalam, S.; Sarkar, A.; Rakshit, A.; Jain, S.; Kumar, A.; Datta, A. Tuning Macrocycles to Design “turn-on” Fluorescence Probes for Manganese(II) Sensing in Live Cells. Chem. Commun. 2015, 51 (13), 2605-2608.

(5) Yadav, A.; Paul, S.; Venkatramani, R.; Ainavarapu, S. R. K. Differences in the Mechanical Unfolding Pathways of Apo- and Copper-Bound Azurins. Sci. Rep. 2018, 8 (1), 1989.

(6) Ramreddy, T.; Kombrabail, M.; Krishnamoorthy, G.; Rao, B. J. Site-Specific Dynamics in TAT Triplex DNA as Revealed by Time-Domain Fluorescence of 2-Aminopurine. J. Phys. Chem. B 2009, 113 (19), 6840-6846. 